\title{
Mixture of lindley and inverse Weibull distributions: properties and estimation
}

\author{
A.S. Al-MoisheER ${ }^{1}$, A.F. DAGHESTANi ${ }^{2}$, K.S. Sultan ${ }^{3}$ \\ ${ }^{1}$ Department of Mathematics, College of Science, Jouf University, \\ P.O.Box 848, Sakaka 72351, SAUDi ARABIA \\ ${ }^{2}$ Department of of Mathematics, College of Science and Humanities - Jubail, Imam Abdulrahman Bin \\ Faisal University, P.O. Box 1982, Dammam 31441, SAUDI ARABIA \\ ${ }^{3}$ Department of Statistics and Operations Research, College of Science, King Saud University, \\ P.O.Box 2455, Riyadh 11451, SAUDI ARABIA
}

\begin{abstract}
In this paper, we talk about a mixture of one-parameter Lindley and inverse Weibull distributions (MLIWD). First, We introduce and discuss the MLIWD. Then, we study the main statistical properties of the proposed mixture and provide some graphs of both the density and the associated hazard rate functions. After that, we estimate the unknown parameters of the proposed mixture via two estimation methods, namely, the generalized method of moments and maximum likelihood. In addition, we compare the estimation methods via some simulation studies to determine the efficacy of the two estimation methods. Finally, we evaluate the performance and behavior of the proposed mixture with different numerical examples and real data application in survival analysis.
\end{abstract}

Key-Words:- Two-component mixture; properties; GMMEs; MLEs.

Received: November 30, 2020. Revised: March 9, 2021. Accepted: March 30, 2021. Published: April 20, 2021.

\section{Introduction}

In the past few decades, mixture models have been recognized as an appropriate model for describing different types of data in many applications such as engineering, reliability studies, life testing problems and many other fields. In general, a mixture component is made by combining two or more components using weights which must add up to one. Here, we propose a mixture of one-parameter Lindley and inverse Weibull distributions as an example of the mixture that are used in real-life applications. It is well known that Lindley distribution (LD) is important in life-time data and reliability. Similarly, inverse Weibull distribution (IWD) is used to model a variety of both reliability and failure data from life testing. Therefore, a mixture of Lindley and inverse Weibull distributions (MLIWD) is proposed. Lindley distribution was proposed by Lindley (1958) in the context of Fiducial and Bayesian statistics to illustrate the difference between fiducial and posterior distributions. Lindley distribution was studied by [1] with various properties and applications. Recently, the mixture of two one-parameter Lindley distributions are proposed in [2] with applications in life testing and also, the mixture of Lindley and Weibull distributions is applied in carbon fibers and in a sample of bladder patients, see [3]. Inverse Weibull distribution, has received attention in the literature over the years. IWD is introduced in reliability data application in [4]. A mixture of TIWD with a graph and hazard rate are discussed in [5]. In addition, the cumulative and the accumulated hazard function are discussed in [6]. The organized of the paper is as follows: in Section 1 we introduce a MLIWD, and discuss previous related studies of these distributions. In Section 2, a MLIWD is familiarized and discussed. Section 3 contains the statistical properties of the proposed mixture LIWD with some graphs of the density and hazard rate functions. In Section 4, we estimate the unknown parameters of the underlying mixture LIWD using the generalized method of moments and the maximum likelihood method. A simulation study of the proposed mixture LIWD is discussed in Section 5. In addition, we calculate some measures from the point estimation of the MLIWD. Finally, in 
Section 6 we apply the proposed mixture model to real dataset in survival analysis and note our conclusion in Section 7.

\section{Proposed Mixture Model}

A MLIWD is considered in this section. We present the mathematical formulation of the mixture with some graphs of the pdf according to the unimodal and bimodal cases. However, we illustrate some statistical properties of the proposed MLIWD. The pdf of the MLIWD takes the form

$$
\begin{aligned}
& f(x ; p, \theta, \lambda, k) \\
& =p\left[\frac{\theta^{2}}{\theta+1}(1+x) e^{-\theta x}\right] \\
& +(1-p)\left[\frac{k}{\lambda^{k}} x^{-(k+1)} e^{-(\lambda x)^{-k}}\right] ; 0 \\
& <p<1 ; x>0 ; \theta, \lambda, k \\
& >0,
\end{aligned}
$$

where $p$ is the mixing parameter (proportion). The first component in (1) considers the pdf of a LD with one parameter $\theta$, while the second component shows the pdf of an IWD with shape parameter $k$ and scale parameter $\lambda$. Thus, the cdf of the MLIWD can be found as follows

$$
\begin{gathered}
F(x ; p, \theta, \lambda, k)=p F_{1}(x ; \theta)+(1 \\
-p) F_{2}(x ; \lambda, k) \\
=p\left[1-\frac{(\theta+1+\theta x) e^{-\theta x}}{\theta+1}\right]+(1 \\
-p)\left[e^{-(\lambda x)^{-k}}\right] ; x>0, \\
\theta, \lambda, k>0 ; 0<p<1 .
\end{gathered}
$$

where $F_{1}(x ; \theta)$ is the cdf of LD with one parameter $\theta$ and $F_{2}(x ; \lambda, k)$ is the cdf of IWD with two parameters $(\lambda, k)$. Figure 1 show different shapes of the MLIWD according to unimodal and bimodal cases. (a)

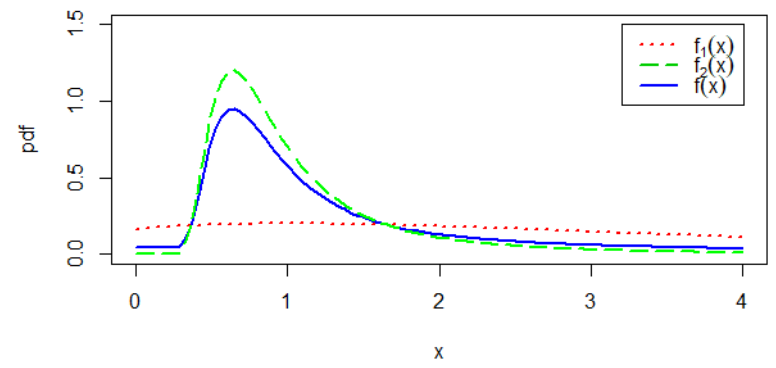

(b)

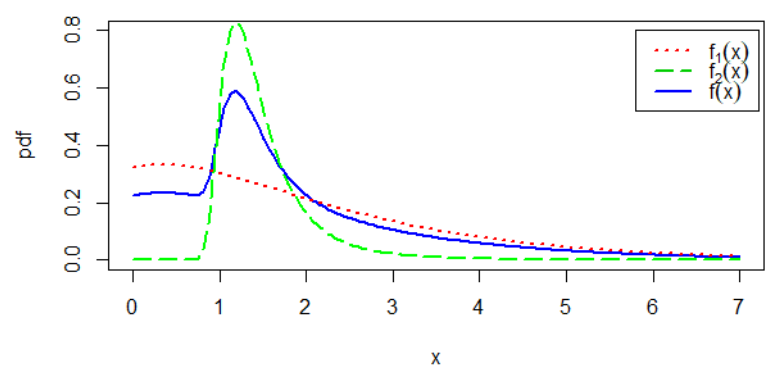

Figure 1: pdf plot of MLIWD with (a) $0=(p=$ $0.25, \theta=0.5, \lambda=0.75, k=2.25) \quad($ b) $\quad$ ? $=(p=$ $0.7, \theta=0.75, \lambda=1.25, k=4.25)$.

\section{Properties of the MLIWD}

In this section, we explain the main statistical properties of the MLIWD and study the behavior of the hazard rate function (HRF) as $x$ tends to zero or infinity with some graphs of the HRF according to the unimodal and bimodal cases. It should be noted that the discussed properties include (the mean, variance, mode, median, skewness (Sk) and kurtosis (Kur), reliability and HRF).

\section{Mean and Variance}

The mean of the MLIWD can be obtained

$$
\begin{aligned}
& E(X)=p\left(\frac{\theta+2}{\theta(\theta+1)}\right) \\
& +(1-p) \lambda^{-1} \Gamma\left(1-\frac{1}{k}\right) ; x \\
& >0 ; 0<p<1 ; \theta, \lambda>0 ; \mathrm{k}>1,
\end{aligned}
$$$$
\text { (3) }
$$

while the variance is found by

$$
\begin{aligned}
& \mathrm{V}(\mathrm{X})=p\left(\frac{2(\theta+3)}{\theta^{2}(\theta+1)}-p\left(\frac{\theta+2}{\theta(\theta+1)}\right)^{2}\right)+(1- \\
& p)\left(\lambda^{-2} \Gamma\left(1-\frac{2}{k}\right)-(1-p) \lambda^{-2} \Gamma^{2}\left(1-\frac{1}{k}\right)\right)- \\
& 2 p(1-p)\left(\frac{\theta+2}{\theta(\theta+1)}\right) \lambda^{-1} \Gamma\left(1-\frac{1}{k}\right) ; x> \\
& 0 ; \quad \theta, \lambda>0 ; \quad k>2 .
\end{aligned}
$$




\section{Mode and Median}

The modes of the proposed mixture are obtained by solving the nonlinear equation below with respect to $x$

$$
\frac{p \theta^{2} e^{-\theta x}}{(\theta+1)}(1-\theta(1+x))+\frac{(1-p) k e^{-(\lambda x)^{-k}}}{\lambda^{k}} x=
$$

0 .

Based on the cdf of the proposed mixture model, we obtain the median of the MLIWD by solving the equation below with respect to $x$

$F(x ; p, \theta, \lambda, k)=p F_{1}(x ; \theta)+(1-$

p) $F_{2}(x ; \lambda, k)=0.5$,

where the first component $F_{1}(x ; \theta)$ considers the cdf of the LD with parameter $\theta$, while the second component $F_{2}(x ; \lambda, k)$ is the cdf of the IWD with parameters $\lambda$ and $k$, as given in (2). Table 1 shows the mode and median of the MLIWD with different choices of parameter according to the unimodal and bimodal cases.

Table 1: The mode(s) and median of the MLIWD

\begin{tabular}{|c|l|l|l|l|l|l|}
\hline Modality & $\boldsymbol{p}$ & $\theta$ & $\boldsymbol{\lambda}$ & $\boldsymbol{k}$ & Mode(s) & Median \\
\hline Unimodal & 0.25 & 0.5 & 0.75 & 2.25 & 1.1318 & 1.6920 \\
\cline { 2 - 7 } case & 0.55 & 0.5 & 0.75 & 2.25 & 1.1304 & 1.9425 \\
\cline { 2 - 7 } & 0.75 & 0.5 & 0.75 & 2.25 & 1.1276 & 2.2100 \\
\cline { 2 - 7 } & 0.95 & 0.5 & 0.75 & 2.25 & 1.1082 & 2.5589 \\
\hline Bimodal & 0.70 & 0.75 & 0.5 & 4.25 & 0.3333 & 1.9479 \\
case & & & & & 1.2215 & \\
\cline { 2 - 7 } & 0.70 & 0.75 & 0.75 & 4.25 & 0.3333 & 1.5213 \\
& & & & & 0.7796 & \\
\cline { 2 - 7 } & 0.70 & 0.75 & 1.00 & 4.25 & 0.3333 & 1.2884 \\
& & & & & 0.5664 & \\
\cline { 2 - 7 } & 0.70 & 0.75 & 1.25 & 4.25 & 0.3333 & 1.1471 \\
& & & & & 0.4404 & \\
\hline
\end{tabular}

We note that from Table 1, the modes and median are affected by changes in $p$ and $\lambda$ according to both the unimodal and bimodal cases. The modes and median decrease as scale parameter $\lambda$ increases. However, when mixing proportion $p$ increases, then the modes decrease while the median values increase.

\section{Measures of Skewness (Sk) and Kurtosis (Kur)}

The Sk coefficient describes the degree of asymmetry of a distribution around its mean, while the Kur coefficient measures the flatness of the distribution. Using the central moments, the Sk and Kur coefficients can be derived. The rth moment about the origin of the MLIWD is equal to

$$
\begin{aligned}
\mu_{r}{ }^{\prime}=p\left(\frac{r !(\theta+r+1)}{\theta^{r}(\theta+1)}\right) & \\
& +(1-p) \frac{1}{\lambda^{r}} \Gamma\left(1-\frac{r}{k}\right), \quad r \\
& =1,2, \ldots . . ;
\end{aligned}
$$

Then we get the Sk and Kur, respectively, as follows

$$
S k=\frac{\mu_{3}}{\sigma^{3}}, \quad \text { Kur }=\frac{\mu_{4}}{\sigma^{4}},
$$

where $\mu_{3}=2 \mu^{3}-3 \mu_{2}{ }^{\prime} \mu+\mu_{3}{ }^{\prime}, \mu_{4}=-3 \mu^{4}+$ $6 \mu_{2}^{\prime} \mu^{2}-4 \mu_{3}{ }^{\prime} \mu+\mu_{4}^{\prime} \quad$ and $\sigma^{3}=(\operatorname{Var}(X))^{\frac{3}{2}}$ Table 2 shows some values of the Sk and Kur based on the unimodal and bimodal cases of the proposed MLIWD.

Table 2: Skewness and kurtosis for MLIWD

\begin{tabular}{|c|l|l|l|l|l|l|}
\hline Modality & $\boldsymbol{p}$ & $\theta$ & $\boldsymbol{\lambda}$ & $\boldsymbol{k}$ & Sk & Kur \\
\hline Unimodal & 0.25 & 0.5 & 0.75 & 2.25 & 1.8501 & 5.1293 \\
\cline { 2 - 7 } case & 0.55 & 0.5 & 0.75 & 2.25 & 0.8743 & 6.3589 \\
\cline { 2 - 7 } & 0.75 & 0.5 & 0.75 & 2.25 & 0.0181 & 6.7651 \\
\hline \multirow{2}{*}{$\begin{array}{c}\text { Bimodal } \\
\text { case }\end{array}$} & 0.70 & 0.75 & 0.75 & 4.25 & 2.0062 & 10.3668 \\
\cline { 2 - 7 } & 0.70 & 0.75 & 1.00 & 4.25 & 2.0712 & 9.4315 \\
\cline { 2 - 7 } & 0.70 & 0.75 & 1.25 & 4.25 & 2.0690 & 8.9980 \\
\hline
\end{tabular}

It also shows that the MLIWD is right or positively Sk. However, it can be seen that the Sk and Kur are affected significantly by changes in the $p$ and $\lambda$ parameters according to the unimodal and bimodal cases. The Kur values increase when mixing proportion $p$ increases and they decrease when scale parameter $\lambda$ increases. Moreover, one value of the $\mathrm{Sk}$ is close to zero, which indicates it is somehow close to the standard normal.

\section{Reliability (R) and Failure Rate Functions (HRF)}

Here, we illustrates the reliability and hazard rate functions of the MLIWD. We study the behavior of the HRF as $x$ tends to zero or infinity with some plots of the HRF according to the unimodal and bimodal cases. Therefore, the reliability and hazard rate functions for the MLIWD can be obtained, respectively, as given below

$$
\begin{aligned}
R(x ; p, \theta, \lambda, k) & =p R_{1}(x ; \theta) \\
& +(1-p) R_{2}(x ; \lambda, k) \\
& =p\left[\frac{(\theta+1+\theta x) e^{-\theta x}}{\theta+1}\right] \\
& +(1-p)\left[1-e^{-(\lambda x)^{-k}}\right] ; x \\
& >0 ; \theta, \lambda, k>0,
\end{aligned}
$$


$r(x ; p, \theta, \lambda, k)=\frac{p f_{1}(x ; \theta)+(1-p) f_{2}(x ; \lambda, k)}{p R_{1}(x ; \theta)+(1-p) R_{2}(x ; \lambda, k)}=$

$\frac{p\left[\frac{\theta^{2}}{\theta+1}(1+x) e^{-\theta x}\right]+(1-p)\left[\frac{k}{\lambda^{k}} x^{-(k+1)} e^{-(\lambda x)^{-k}}\right]}{p\left[\frac{(\theta+1+\theta x) e^{-\theta x}}{\theta+1}\right]+(1-p)\left[1-e^{-(\lambda x)^{-k}}\right]}$.

Then, the HRF of the MLIWD satisfies the limits, as illustrated in Lemma below.

\section{Lemma}

$$
\lim _{x \rightarrow 0} r(x ; p, \theta, \lambda, k)=\frac{p \theta^{2}}{\theta+1},
$$

and

$$
\lim _{x \rightarrow \infty} r(x ; p, \theta, \lambda, k)=0
$$

\section{Proof}

Applying the limit concept to (11), we get

$\lim _{x \rightarrow 0} r(x ; p, \theta, \lambda, k)=$

$\lim _{x \rightarrow 0} \frac{p\left[\frac{\theta^{2}}{\theta+1}(1+x) e^{-\theta x}\right]+(1-p)\left[\frac{k}{\lambda^{k}} x^{-(k+1)} e^{-(\lambda x)^{-k}}\right]}{p\left[\frac{(\theta+1+\theta x) e^{-\theta x}}{\theta+1}\right]+(1-p)\left[1-e^{-(\lambda x)^{-k}}\right]}$
$=\lim _{x \rightarrow 0} \frac{A+B}{C+D}=\lim _{x \rightarrow 0} \frac{A}{C+D}+\lim _{x \rightarrow 0} \frac{B}{C+D}$,

where

$A=p\left[\frac{\theta^{2}}{\theta+1}(1+x) e^{-\theta x}\right], B=(1-$

p) $\left[\frac{k}{\lambda^{k}} x^{-(k+1)} e^{-(\lambda x)^{-k}}\right], C=p\left[\frac{(\theta+1+\theta x) e^{-\theta x}}{\theta+1}\right]$ and $D=(1-p)\left[1-e^{-(\lambda x)^{-k}}\right]$. Therefore, we solve the first part of (13) as follows

$\lim _{x \rightarrow 0} \frac{A}{C+D}$

$=\lim _{x \rightarrow 0} \frac{p\left[\frac{\theta^{2}}{\theta+1}(1+x) e^{-\theta x}\right]}{p\left[\frac{(\theta+1+\theta x) e^{-\theta x}}{\theta+1}\right]+(1-p)\left[1-e^{-(\lambda x)^{-k}}\right]}$

$=\frac{\frac{p \theta^{2}}{\theta+1}}{p+(1-p)}=\frac{p \theta^{2}}{\theta+1}$.

To solve the second part of (13) we need to divide $\frac{B}{C+D}$ by $\frac{B}{C+D}$ and take the limit as given below

$\lim _{x \rightarrow 0} \frac{\frac{(1-p) k}{\lambda^{k}}}{\frac{e^{(\lambda x)^{-k}} p}{x^{-(k+1)}}\left[\frac{(\theta+1+\theta x) e^{-\theta x}}{\theta+1}\right]+\frac{e^{(\lambda x)^{-k}(1-p)}}{x^{-(k+1)}}\left[1-e^{-(\lambda x)^{-k}}\right]}=$

$\frac{\frac{(1-p) k}{\lambda^{k}}}{\infty}=0$.

Hence, by substituting (14) and (15) in (13), the first part of Lemma is proven when $x$ tends to zero. Now, to prove (12) in Lemma we need to solve the limit as $x$ tends to $\infty$. So, we divide the HRF $r(x ; p, \theta, \lambda, k)$ by $e^{-\theta x}$ and solve the limit using some of the limit properties as follows $\lim _{x \rightarrow \infty} r(x ; p, \theta, \lambda, k)$

$=\lim _{x \rightarrow \infty} \frac{\frac{p \theta^{2}}{\theta+1}(1+x)+\frac{(1-p) k}{\lambda^{k}} x^{-(k+1)} e^{-(\lambda x)^{-k}+\theta x}}{p\left[\frac{(1+\theta(1+x))}{\theta+1}\right]+(1-p)\left[1-e^{-(\lambda x)^{-k}+\theta x}\right]}$

$=\lim _{x \rightarrow \infty} \frac{A+B}{C+D}=\lim _{x \rightarrow \infty} \frac{A}{C+D}+\lim _{x \rightarrow \infty} \frac{B}{C+D}$.

In (16) we assume that $A=\frac{p \theta^{2}}{\theta+1}(1+x), B=$ $\frac{(1-p) k}{\lambda^{k}} x^{-(k+1)} e^{-(\lambda x)^{-k}+\theta x}, C=p\left[\frac{(1+\theta(1+x))}{\theta+1}\right]$ and $D=(1-p)\left[1-e^{-(\lambda x)^{-k}+\theta x}\right]$. Now, to solve the first limit in (16) we divide the quantity $\frac{A}{C+D}$ by $(1+x)$ and then solve the limit when $x \rightarrow \infty$. Knowing that, $\quad \lim _{x \rightarrow \infty} D=\lim _{x \rightarrow \infty} p_{2}[1-$ $\left.e^{-(\lambda x)^{-k}+\theta x}\right]=-\infty$ since $-(\lambda x)^{-k}+\theta x>0$ and if $x \rightarrow \infty$ then $-(\lambda x)^{-k}+\theta x \rightarrow \infty$. Thus, $\lim _{x \rightarrow \infty} \frac{A}{C+D}=\lim _{x \rightarrow \infty} \frac{\frac{p \theta^{2}}{\theta+1}}{\frac{p}{(\theta+1)(1+x)}+\frac{p \theta}{(\theta+1)}-\infty}=0$.

Next, we use the following definition to solve the second limit in (16).

\section{Definition}

Let $f(x), g(x)$ be functions defined in a reduced neighborhood of $a$, where $a$ is a real number $\infty$ or $\infty$. Then, we say that $f \cong g$ at $a$ if $\lim _{x \rightarrow a}\left(\frac{f(x)}{g(x)}\right)=1$. Now for the sake of simplicity, we multiply $B=$ $\frac{(1-p) k}{\lambda^{k}}\left(\frac{x^{-k}}{x}\right) e^{-(\lambda x)^{-k}+\theta x}$ by $\frac{-\lambda^{-k}}{-\lambda^{-k}}$ to make the limit easy to solve using an equivalent function. Thus, we have $B=\frac{(1-p) k}{\lambda^{k}}\left(\frac{1}{-\lambda^{-k}}\right) \frac{(-\lambda x)^{-k}}{x} e^{-(\lambda x)^{-k}+\theta x}=$ $\left(\frac{-(1-p) k}{x}\right)(-\lambda x)^{-k} e^{-x^{-k}\left[\lambda^{-k}-\frac{\theta}{\left.x^{-(k+1)}\right]}\right.}$. Suppose $f(x)=\lambda^{-k}-\frac{\theta}{x^{-(k+1)}} \quad$ and $g(x)=\lambda^{-k}$, then $\lim _{x \rightarrow \infty}\left(\frac{f(x)}{g(x)}\right)=\lim _{x \rightarrow \infty}\left(\frac{\lambda^{-k}-\frac{\theta}{x^{-(k+1)}}}{\lambda^{-k}}\right)=\lim _{x \rightarrow \infty}(1-$ $\left.\frac{\theta}{\lambda^{-k} x^{-(k+1)}}\right)=1$, which implies that $f \cong g$ and we get

$$
e^{-x^{-k}\left[\lambda^{-k}-\frac{\theta}{x^{-(k+1)}}\right]} \cong e^{-(\lambda x)^{-k}}
$$

Therefore, from (18) we have

$$
\begin{aligned}
& \lim _{x \rightarrow \infty} B \\
& =\lim _{x \rightarrow \infty}[-(1 \\
& \left.-p) k\left(\frac{1}{x}\right)\right] \lim _{x \rightarrow \infty}\left[-(\lambda x)^{-k} e^{-(\lambda x)^{-k}}\right]=0,
\end{aligned}
$$

and 


$$
\lim _{x \rightarrow \infty} \frac{B}{C+D}=\frac{0}{\infty}=0 .
$$

By substituting (17) and (19) in (16), we prove the required limit in Lemma (3.1). Figure 2 (a-b) shows two cases of the HRF according to the unimodal and bimodal cases for the MLIWD. Further, we have shown that the HRF values are limited by the interval $\left(\frac{p \theta^{2}}{\theta+1}, 0\right)$. To study the behavior of the HRF curve, two cases arise as follows:

In unimodal case, the HRF curve increases in $\left(0, x_{1}^{*}\right)$ until it arrives the peak of the curve at $x_{1}^{*}$ (mode value) [see Figure 2(a)]. Then, the curve decreases gradually in the interval $\left(x_{1}^{*}, x_{2}^{*}\right)$ and eventually assumes a value of zero when $x_{2}^{*} \rightarrow \infty$.

In the bimodal case, the HRF curve increases until it reaches the value of the first peak in the interval $\left(0, x_{1}^{*}\right)$, while it decreases in the period $\left(x_{1}^{*}, x_{2}^{*}\right)$. Next, the curve increases slowly to form a second peak at $x_{3}^{*}$ in $\left(x_{2}^{*}, x_{3}^{*}\right)$. After the curve has reached all the peaks or mode values, the curve decreases gradually in the interval $\left(x_{3}^{*}, x_{4}^{*}\right)$ until it reaches zero as $x_{4}^{*} \rightarrow \infty$ [see Figure 2(b)]. We also noticed that the first top of the curve has a higher peak than the second top.

(a)

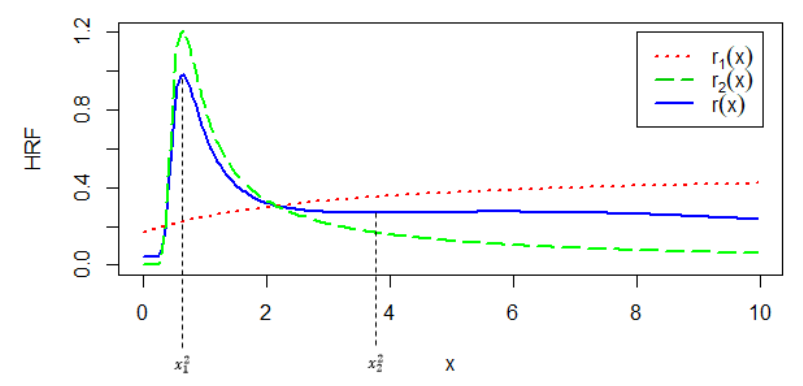

(b)

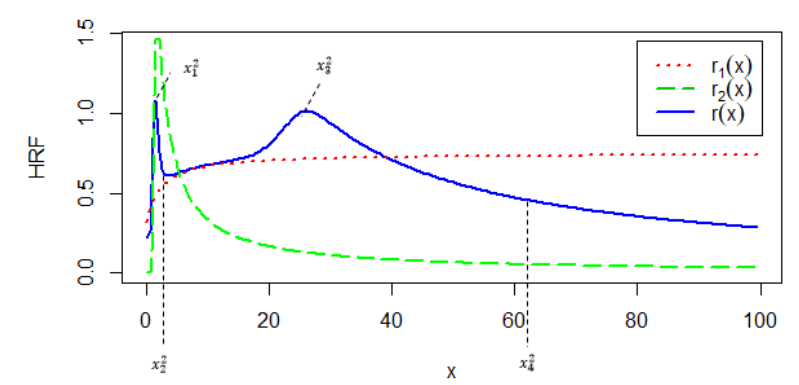

Figure 2: HRF plot of MLIWD with (a) $?=(p=$ $0.25, \theta=0.5, \lambda=0.75, k=2.25) \quad(\mathrm{b}) \quad$ ? $=(p=$ $0.7, \theta=0.75, \lambda=1.25, k=4.25)$.

\section{Estimation}

This section discuss two methods to estimate the parameters of the proposed MLIWD, namely, the generalized method of moments and the maximum likelihood method denoted by GMM and MLM, respectively.

\subsection{Generalized Method of Moments}

Here, we present the generalized method of moments (GMM) estimators for the parameters $(p, \theta, \lambda, k)$ of the MLIWD by using the moment conditions and the definition of GMM estimators [see Hall (2005)]. First, we need to write the moment conditions of the MLIWD as follows

$x_{i}-p \frac{\theta+2}{\theta(\theta+1)}+(1-p) \lambda^{-1} \Gamma\left(1-\frac{1}{k}\right)=$

$0, \quad k>1,(21)$

$x_{i}^{2}-p \frac{2(\theta+3)}{\theta^{2}(\theta+1)}+(1-p) \lambda^{-2} \Gamma\left(1-\frac{2}{k}\right)=$

$0, k>2$,

$x_{i}^{3}-p \frac{6(\theta+4)}{\theta^{3}(\theta+1)}+(1-p) \lambda^{-3} \Gamma\left(1-\frac{3}{k}\right)=$

$0, k>3$, (23)

and

$x_{i}^{4}-p \frac{24(\theta+5)}{\theta^{4}(\theta+1)}+(1-p) \lambda^{-4} \Gamma\left(1-\frac{4}{k}\right)=$

$0 ; i=1,2, \ldots, n ; k>4$. (24)

Now, to get the GMM estimators we must discover the parameter values that minimize the coast function $Q_{n}(\Theta), \Theta=(p, \theta, \lambda, k)$ of the MLIWD, as given below

$$
\begin{aligned}
& Q_{n}(\Theta) \\
& =n^{-1} \sum_{i=1}^{n}\left[x_{i}-E(X) \quad x_{i}^{2}-E\left(X^{2}\right) \quad x_{i}^{3}-E\left(X^{3}\right) \quad x_{i}^{4}-E\left(X^{4}\right)\right]^{\prime} \\
& \times\left[\begin{array}{llll}
1 & 0 & 0 & 0 \\
0 & 1 & 0 & 0 \\
0 & 0 & 1 & 0 \\
0 & 0 & 0 & 1
\end{array}\right] n^{-1} \sum_{i=1}^{n}\left[\begin{array}{c}
x_{i}-E(X) \\
x_{i}^{2}-E\left(X^{2}\right) \\
x_{i}^{3}-E\left(X^{3}\right) \\
x_{i}^{4}-E\left(X^{4}\right)
\end{array}\right] \\
& =\left(\frac{\sum_{i=1}^{n} x_{i}}{n}\right)^{2}-\left(\frac{2 \sum_{i=1}^{n} x_{i}}{n}\right) E(X)+(E(X))^{2}+ \\
& \left(\frac{\sum_{i=1}^{n} x_{i}^{2}}{n}\right)^{2}-\left(\frac{2 \sum_{i=1}^{n} x_{i}^{2}}{n}\right) E\left(X^{2}\right) \\
& +\left(E\left(X^{2}\right)\right)^{2}+\left(\frac{\sum_{i=1}^{n} x_{i}^{3}}{n}\right)^{2}-\left(\frac{2 \sum_{i=1}^{n} x_{i}^{3}}{n}\right) E\left(X^{3}\right)+ \\
& \left(E\left(X^{3}\right)\right)^{2}+\left(\frac{\sum_{i=1}^{n} x_{i}^{4}}{n}\right)^{2} \\
& -\left(\frac{2 \sum_{i=1}^{n} x_{i}^{4}}{n}\right) E\left(X^{4}\right)+\left(E\left(X^{4}\right)\right)^{2} \text {, }
\end{aligned}
$$

where

$$
\begin{aligned}
& E(X)=p \frac{\theta+2}{\theta(\theta+1)}+(1-p) \lambda^{-1} \Gamma\left(1-\frac{1}{k}\right), \\
& E\left(X^{2}\right)=p \frac{2(\theta+3)}{\theta^{2}(\theta+1)}+(1-p) \lambda^{-2} \Gamma\left(1-\frac{2}{k}\right), \\
& E\left(X^{3}\right)=p \frac{6(\theta+4)}{\theta^{3}(\theta+1)}+(1-p) \lambda^{-3} \Gamma\left(1-\frac{3}{k}\right),
\end{aligned}
$$

and

$$
E\left(X^{4}\right)=p \frac{24(\theta+5)}{\theta^{4}(\theta+1)}+(1-p) \lambda^{-4} \Gamma\left(1-\frac{4}{k}\right) .
$$


Next, we differentiate (21-24) with respect to each component of $(p, \theta, \lambda, k)$ and equate these derivative equations to zeroto obtain the GMM estimators. Therefore, we have the following system of nonlinear equations

$$
\begin{aligned}
& \frac{\partial Q_{n}(\Theta)}{\partial p}=2\left[\left(\frac{(1-p) \Gamma\left(1-\frac{4}{k}\right)}{\lambda^{4}}+\right.\right. \\
& \left.\left.\frac{24 p(5+\theta)}{\theta^{4}(1+\theta)}\right)\left(\frac{24(5+\theta)}{\theta^{4}(1+\theta)}-\frac{\Gamma\left(1-\frac{4}{k}\right)}{\lambda^{4}}\right)\right]=0, \quad(26) \\
& \frac{\partial Q_{n}(\Theta)}{\partial \theta}=48 p\left[\left(\frac{(1-p) \Gamma\left(1-\frac{4}{k}\right)}{\lambda^{4}}+\right.\right. \\
& \left.\left.\frac{24 p(5+\theta)}{\theta^{4}(1+\theta)}\right)\left(\frac{1}{\theta^{4}(1+\theta)}-\left(\frac{\theta^{3}(4+5 \theta)(5+\theta)}{\left(\theta^{4}(1+\theta)\right)^{2}}\right)\right)\right]=0,
\end{aligned}
$$

$$
\frac{\partial Q_{n}(\Theta)}{\partial \lambda}=-8(1-p)\left[\left(\frac{(1-p) \Gamma\left(1-\frac{4}{k}\right)}{\lambda^{4}}+\right.\right.
$$$$
\left.\left.\frac{24 p(5+\theta)}{\theta^{4}(1+\theta)}\right)\right]\left[\frac{\Gamma\left(1-\frac{4}{k}\right)}{\lambda^{5}}\right]=0,(28)
$$$$
\frac{\partial Q_{n}(\Theta)}{\partial k}=8(1-p)\left[\left(\frac{(1-p) \Gamma\left(1-\frac{4}{k}\right)}{\lambda^{4}}+\right.\right.
$$$$
\left.\left.\frac{24 p(5+\theta)}{\theta^{4}(1+\theta)}\right) \psi\left(1-\frac{4}{k}\right)\left(\frac{\Gamma\left(1-\frac{4}{k}\right)}{\lambda^{4} k^{2}}\right)\right]=0,
$$

where $\psi$ is the digamma function. Finally, we solve the system of equations (26-29) and get the GMM estimates of the underlying model parameters. We use the (gmm) package in R to solve the systems and the numerical results are shown later in Section 5.

\subsection{Maximum Likelihood Estimation}

The MLE is used in a wide range of statistical analyses. In this subsection, we obtain the MLEs for the unknown parameters of the MLIWD by finding the parameters that maximize the likelihood function, given the observations. So, let $x_{1}, x_{2}, \ldots \ldots \ldots, x_{n}$ be a random sample from the MLIWD, then the loglikelihood function is given as

$$
\begin{aligned}
& L^{*}=\log L=\sum_{i=1}^{n} \log \left(p \frac{\theta^{2}}{\theta+1}\left(1+x_{i}\right) e^{-\theta x_{i}}+\right. \\
& \left.(1-p) \frac{k}{\lambda^{k}} x_{i}^{-(k+1)} e^{-\left(\lambda x_{i}\right)^{-k}}\right) ; x>0, \theta, \lambda, k>0, \\
& 0<p<1 .
\end{aligned}
$$

Now, by differentiating the log-likelihood function in (30) with respect to each parameters $(p, \theta, \lambda, k)$ we get the first order derivatives of $L^{*}$. Next, by equating these derivative equations to zero and solving this system of equations, we obtain the MLEs of the four unknown parameters of the proposed MLIWD. The system of equations is shown below

$$
\frac{\partial L^{*}}{\partial p}=\sum_{i=1}^{n} \frac{f_{1}\left(x_{i} ; \theta\right)-f_{2}\left(x_{i} ; \lambda, k\right)}{p f_{1}\left(x_{i} ; \theta\right)+(1-p) f_{2}\left(x_{i} ; \lambda, k\right)}=0 \text { ， }
$$

$$
\frac{\partial L^{*}}{\partial \theta}=\sum_{i=1}^{n} \frac{p\left(1+x_{i}\right) e^{-\theta x_{i}}\left[\frac{\theta(2+\theta)}{(\theta+1)^{2}}-x_{i} \frac{\theta^{2}}{(\theta+1)}\right]}{p f_{1}\left(x_{i} ; \theta\right)+(1-p) f_{2}\left(x_{i} ; \lambda, k\right)}=0,
$$

$\frac{\partial L^{*}}{\partial \lambda}=$

$\sum_{i=1}^{n} \frac{(1-p) k x_{i}^{-(k+1)} e^{-\left(\lambda x_{i}\right)^{-k}\left[k x_{i}^{-k} \lambda^{-(2 k+1)}-k \lambda^{-(k+1)}\right]}}{p f_{1}\left(x_{i} ; \theta\right)+(1-p) f_{2}\left(x_{i} ; \lambda, k\right)}=$

0 , (33)

$\frac{\partial L^{*}}{\partial k}=\sum_{i=1}^{n} \frac{(1-p) x_{i}^{-(k+1)} e^{-\left(\lambda x_{i}\right)^{-k}}}{p f_{1}\left(x_{i} ; \theta\right)+(1-p) f_{2}\left(x_{i} ; \lambda, k\right)} \times$
$\left[\frac{\lambda^{k}(1-k \log \lambda)}{\lambda^{2 k}}-\frac{k \log x_{i}}{\lambda^{k}}+\frac{k\left(\lambda x_{i}\right)^{-k} \log \left(-\lambda x_{i}\right)}{\lambda^{k}}\right]=$

$0, \quad(34)$

where $f_{1}\left(x_{i} ; \theta\right)$ and $f_{2}\left(x_{i} ; \lambda, k\right)$ are the pdf of the LD and IWD, respectively. We get the ML estimators for the unknown parameters of the proposed MLIWD by solving the system of equations (31-34) by using some $\mathrm{R}$ packages with several numerical techniques to find the maximum value such as the NewtonRaphson and Broyden methods. The results are shown in Section 5.

\section{Simulation Studies}

Here, we perform some simulation studies for the proposed mixture model, including solving the nonlinear equations of the GMM and ML given in Section 4. Moreover, we analyze some statistical measures of the estimates in to examine the performance of the quality of the estimates. Thus, we compute the bias, (MSE) mean squared error and the(RE) relative efficiency of the estimates. In addition, we construct the (CI) confidence interval of the estimated parameters for the MLIWD according to the GMM and ML estimation methods. We also compute the $(\mathrm{CP})$ coverage probability and average length of these estimated intervals. The (CP) is a statistical technique for calculating the amount of time that the (CI) takes up or covering the true value of the parameters. We generate random samples 30 , 50 and 100 in size from the MLIWD model according to the unimodal and bimodal cases. The random samples are generated using the function (rlindley) (rinvweibull) in the R software for the components mixture. We solve the system of nonlinear equations (21-24) for the GMM and (31-34) for the ML methods using some $\mathrm{R}$ software packages such as (gmm) and (nleqslv). All the numerical results for the simulation studies of the MLIWD are shown and discussed in Tables 3 and 4. In Table 3, we conclude that the ML estimate of $p$ is over-estimated in the unimodal and bimodal cases. In addition, the ML 
estimates of $\lambda$ and $k$ are under-estimated according to the unimodal and bimodal cases. Besides, we can see that the estimated bias of the model parameters are over-estimated in some cases and underestimated in other cases according to the estimation method. Also, Table 3 shows that the GMM and ML estimates of the unknown parameters satisfy the consistency, as the MSE decreases as $n$ increases. However, based on the value of the relative efficiency, it can be seen that the GMMEs of the unknown parameters are better than the MLEs, as they have ewer MSEs. In Table 4, we calculate the average length of the confidence intervals based on 1000 replications, and the value of the average length decreases as the sample size increases, as the interval becomes narrower. Further, the average length increases when the confidence level increases and the confidence interval become wider.

\section{Data Analysis}

In this section, we use a real dataset to compare the MLIWD in data fitting versus other mixtures such as a Weibull mixture (WM), a Lindley mixture (LM) and an inverse Weibull mixture (IWM). Application will be considered to show a better fit mixture than other mixtures.

\section{Application}

The dataset considered in this application consists of the survival times (in days) of 72 guinea pigs infected, as reported by [7]. Therefore, we use this real dataset to show that the MLIWD can be a better mixture than other mixtures. Table 5 shows the MLE of the unknown parameters, the AIC and the statistic value of the K-S test with a p-value.

Table 5: Results for Application

\begin{tabular}{|c|c|c|c|}
\hline Model & MLE & AIC & $\begin{array}{l}\text { K-S } \\
\text { (p-value) }\end{array}$ \\
\hline MLIWD & $\begin{array}{l}\hat{p}=0.2994 \\
\hat{\theta}=0.8494 \\
\hat{k}=2.8788 \\
\hat{\lambda}=0.7689\end{array}$ & 192.182 & $\begin{array}{l}0.0652 \\
(0.9195)\end{array}$ \\
\hline MTLD & $\begin{array}{l}\hat{p}=0.4882 \\
\hat{\theta}_{1}=0.8681 \\
\hat{\theta}_{2}=0.8682\end{array}$ & 219.8569 & $\begin{array}{l}0.2466 \\
(0.0003)\end{array}$ \\
\hline MTIWD & $\begin{array}{l}\hat{p}=0.1062 \\
\hat{k}_{1}=1.0557 \\
\hat{\lambda}_{1}=2.7595 \\
\hat{k}_{2}=2.5178\end{array}$ & 196.8675 & $\begin{array}{l}0.0654 \\
(0.9175)\end{array}$ \\
\hline
\end{tabular}

\begin{tabular}{|l|l|l|l|}
\hline & $\hat{\lambda}_{2}=0.7512$ & & \\
\hline MTLWD & $\hat{p}=0.2980$ & & \\
& $\hat{\theta}=0.6834$ & & \\
& $\hat{\beta}=2.6300$ & 197.7033 & 0.0913 \\
& $\hat{\alpha}=1.7227$ & & $(0.5855)$ \\
\hline
\end{tabular}

As can be seen from Table 5, the mixture of the MLIWD fits the data better than the other mixtures according to the AIC criteria and the K-S test. In Figure 3, we have plotted the histogram and the ECDF for the data application.
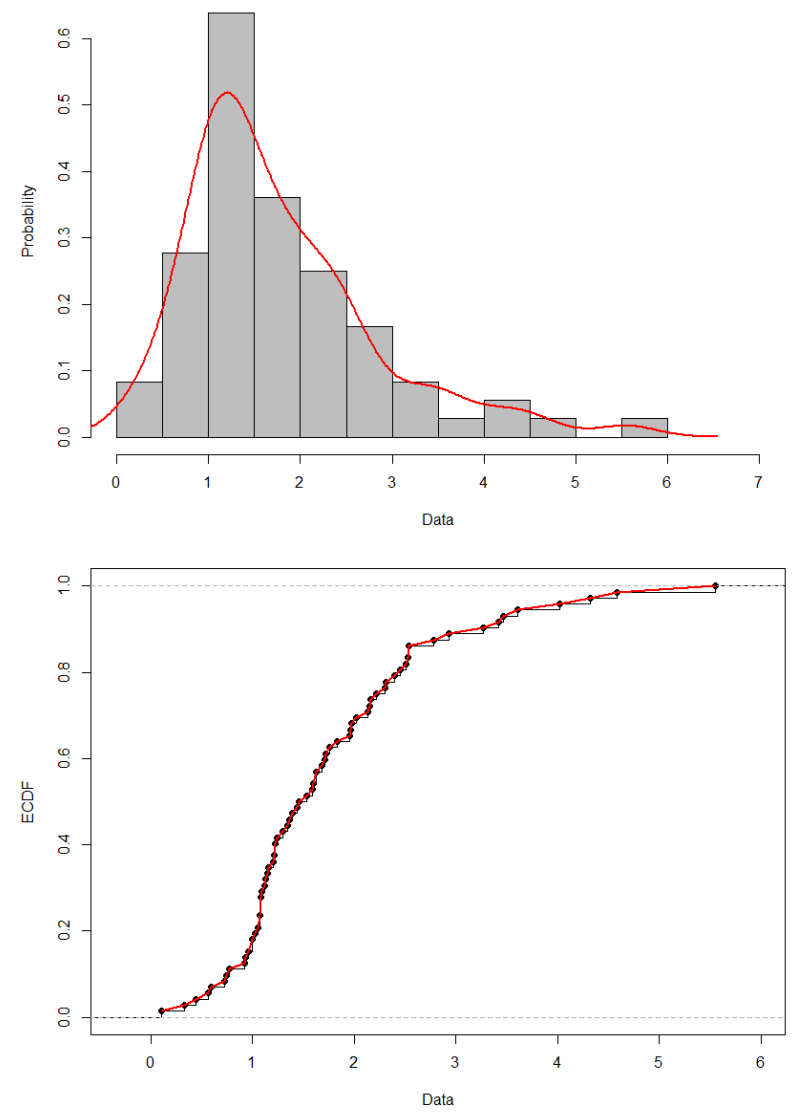

Figure3: Histogram and ECDF plots for application

\section{Conclusion}

In this paper, we proposed a mixture LIWD. The essential statistical properties of the proposed mixture LIWD include the mean, mode, variance, median, measures of Sk and Kur and the behavior of the HRF with some graphs of the pdf and HRF. The GMM and MLM were used to estimate the unknown parameters of the MLIWD. Moreover, some simulation studies were performed in order to explore the performance of the estimares through estimation. In addition, the efficiency of the simulation studies was determined based on the reported MSE and RE values, the average length of the estimated CIs and the percentage of the coverage probability. In 
general, we observed from the simulation results that when the sample size increases, the mean squared error decreases; which satisfies the consistency from which we can see the asymptotically consistent property of the estimates. We also found that the GMM provides better results for estimating model parameters than the ML method according to the MSE and RE values for both the unimodal and bimodal cases. We also presented two different numerical examples for the MLIWD that considered the unimodal and bimodal cases and estimated the unknown parameters of the underlying mixture model with their standard errors using the GMM and ML estimation. Finally, we illustrated a real data application in Survival analysis. We conclude that the MLIWD is a reasonably good model to fit the data from different applications compared to other mixture models such as the MTWD, the MTIWD, the MLWD and the MTLD based on the AIC criteria and the K-S test with a p-value.

\section{References:}

[1] Ghitany, M.E., Atieh, B. and Nadarajah, S. Lindley distribution and its applications, Mathematics and Computers in Simulation, (2008), 78, 493-506.

[2] Al-Moisheer, A. S., Daghestani, A. F. and Sultan, K. S. Mixture of Two One-Parameter Lindley Distributions: Properties and Estimation. Journal of Statistical Theory and Practice, (2021), 15:11, 1-21. https://doi.org/10.1007/s42519-020-001334.

[3] Daghestani, A. F., Sultan, K. S. and AlMoisheer, A. S. Mixture of Lindley and Weibull Distributions: Properties and Estimation., Journal of Statistics Applications \& Probability, (2021), to appear.

http://www.naturalspublishing.com/FC.asp? JorID $=3$.

[4] Keller, A.Z. and Kamath, A.R. Reliability Analysis of CNC Machine Tools, Reliability Engineering, (1982), 3(6), 449-473. DOI:10.1016/0143-8174(82)90036-1 .

[5] Sultan, K.S., Ismail, M.A. and AL-Moisheer, A.S. Mixture of two inverse Weibull distributions: Properties and estimation, Comput. Statist. Dat.Anal., (2007), 51, 5377-5387. doi:10.1016/j.csda.2006.09.016.

[6] Škanata, Dejan. A Note on the Cumulative and the Accumulated Hazard Function International Journal of Circuits, Systems and Signal Processing, (2020), 14, 149-153 doi:10.46300/9106.2020.14.23 .

[7] Bjerkedal, T. Acquisition of Resistance in Guinea Pies infected with Different Doses of Virulent Tubercle Bacilli, American Journal of Hygiene, (1960), 72, 130-48. DOI: 10.1093/oxfordjournals.aje.a120129.

\section{Contribution of individual authors to the creation of a scientific article (ghostwriting policy)}

Author Contributions: Please, indicate the role and the contribution of each author:

Example

John Smith, Donald Smith carried out the simulation and the optimization.

George Smith has implemented the Algorithm 1.1 and 1.2 in $\mathrm{C}++$.

Maria Ivanova has organized and executed the experiments of Section 4.

George Nikolov was responsible for the Statistics.

Follow: www.wseas.org/multimedia/contributor-

role-instruction.pdf

\section{Sources of funding for research presented in a scientific article or scientific article itself \\ Report potential sources of funding if there is any}

\section{Creative Commons Attribution License 4.0 (Attribution 4.0 International, CC BY 4.0)}

This article is published under the terms of the Creative Commons Attribution License 4.0

https://creativecommons.org/licenses/by/4.0/deed.en_US 
Table 3: Bias, MSE and RE of the MLIWD estimated parameters

\begin{tabular}{|c|c|c|c|c|c|c|c|c|c|c|c|c|c|}
\hline \multirow{3}{*}{$\stackrel{\Theta}{=}(p, \theta, \lambda, k)$} & \multirow{3}{*}{$n$} & \multirow{2}{*}{\multicolumn{4}{|c|}{$\frac{\text { GMM }}{\text { Bias \& MSE }}$}} & \multicolumn{4}{|c|}{ ML } & \multirow{2}{*}{\multicolumn{4}{|c|}{$\mathrm{RE}$}} \\
\hline & & & & & & & & as \& $\mathrm{M}$ & & & & & \\
\hline & & $\hat{p}$ & $\hat{\theta}$ & $\hat{\lambda}$ & $\hat{k}$ & $\hat{p}$ & $\hat{\theta}$ & $\hat{\lambda}$ & $\hat{k}$ & $\hat{p}$ & $\hat{\theta}$ & $\hat{\lambda}$ & $\hat{k}$ \\
\hline \multirow{6}{*}{$\begin{array}{c}(0.25,0.5 \\
0.75,2.25)^{*}\end{array}$} & \multirow[t]{2}{*}{30} & 0.5438 & 0.2250 & 0.9776 & -1.1710 & 0.7582 & 0.4586 & $\begin{array}{c}- \\
0.7452\end{array}$ & -2.1387 & \multirow[t]{2}{*}{1.9370} & \multirow[t]{2}{*}{1.4375} & \multirow[t]{2}{*}{5.5852} & \multirow[t]{2}{*}{3.2742} \\
\hline & & 0.2968 & 0.0512 & 0.0998 & 1.3971 & 0.5749 & 0.2272 & 0.5574 & 4.5744 & & & & \\
\hline & \multirow[t]{2}{*}{50} & 0.4777 & 0.2034 & $\begin{array}{c}- \\
0.2611\end{array}$ & 0.3463 & 0.7440 & 0.4172 & $\begin{array}{c}- \\
0.7459\end{array}$ & -2.0697 & \multirow{2}{*}{2.8971} & \multirow{2}{*}{4.5148} & \multirow{2}{*}{7.9063} & \multirow{2}{*}{35.6769} \\
\hline & & 0.2284 & 0.0439 & 0.0704 & 0.1201 & 0.5545 & 0.1982 & 0.5566 & 4.2848 & & & & \\
\hline & \multirow[t]{2}{*}{100} & 0.4373 & $\begin{array}{c}- \\
0.0674\end{array}$ & $\begin{array}{c}- \\
0.1071\end{array}$ & 0.3034 & 0.7423 & 0.3168 & $\begin{array}{c}- \\
0.7159\end{array}$ & -2.0291 & \multirow[t]{2}{*}{2.4133} & \multirow[t]{2}{*}{21.9241} & \multirow[t]{2}{*}{38.9015} & \multirow[t]{2}{*}{44.5119} \\
\hline & & 0.1914 & 0.0079 & 0.0132 & 0.0926 & 0.5512 & 0.1732 & 0.5135 & 4.1218 & & & & \\
\hline \multirow{6}{*}{$\begin{array}{c}(0.70,0.75 \\
1.25 \\
4.25)^{* *}\end{array}$} & \multirow[t]{2}{*}{30} & $\begin{array}{c}- \\
0.0232\end{array}$ & 0.1014 & 0.6156 & -0.5512 & 0.2097 & $\begin{array}{c}- \\
0.6094\end{array}$ & $\begin{array}{c}- \\
1.1954\end{array}$ & -0.7435 & \multirow[t]{2}{*}{1.1147} & \multirow[t]{2}{*}{9.4670} & \multirow[t]{2}{*}{3.2413} & \multirow[t]{2}{*}{1.4675} \\
\hline & & 0.0401 & 0.0454 & 0.4409 & 0.3769 & 0.0447 & 0.4298 & 1.4291 & 0.5531 & & & & \\
\hline & \multirow[t]{2}{*}{50} & $\begin{array}{c}- \\
0.0312\end{array}$ & 0.0659 & 0.6118 & -0.5270 & 0.2003 & $\begin{array}{c}- \\
0.5426\end{array}$ & $\begin{array}{c}- \\
1.1950\end{array}$ & -0.7374 & \multirow{2}{*}{1.3441} & \multirow{2}{*}{15.4879} & \multirow{2}{*}{3.4319} & \multirow{2}{*}{1.6749} \\
\hline & & 0.0311 & 0.0248 & 0.4161 & 0.3251 & 0.0418 & 0.3841 & 1.4280 & 0.5445 & & & & \\
\hline & \multirow[t]{2}{*}{100} & - & 0.0374 & 0.5862 & -0.5058 & 0.1987 & $\begin{array}{c}- \\
0.5243\end{array}$ & $\begin{array}{c}- \\
1.1949\end{array}$ & -0.7349 & \multirow{2}{*}{2.1925} & \multirow[t]{2}{*}{25.4726} & 3.6573 & 1.7802 \\
\hline & & 0.0187 & 0.0146 & 0.3904 & 0.3039 & 0.0410 & 0.3719 & 1.4278 & 0.5410 & & & & \\
\hline
\end{tabular}

*Unimodal case. **Bimodal case.

Relative Efficiency $(\mathrm{RE})=\frac{\boldsymbol{M S E _ { \boldsymbol { M L } } ( \widehat { \boldsymbol { \Theta } } )}}{\boldsymbol{M S E _ { G M M } ( \widehat { \boldsymbol { \Theta } } )}}$ 
Table 4: Average length of the estimated CIs for the MLIWD

\begin{tabular}{|c|c|c|c|c|c|c|c|c|c|c|c|c|c|c|c|c|c|}
\hline \multirow{5}{*}{$\begin{array}{l}\Theta \\
=(p, \theta, \lambda, k)\end{array}$} & \multirow{5}{*}{$n$} & \multicolumn{16}{|c|}{ Estimation Methods } \\
\hline & & \multicolumn{8}{|c|}{ GMM } & \multicolumn{8}{|c|}{ ML } \\
\hline & & \multicolumn{8}{|c|}{ Confidence Level } & \multicolumn{8}{|c|}{ Confidence Level } \\
\hline & & \multicolumn{4}{|c|}{$90 \%$} & \multicolumn{4}{|c|}{$95 \%$} & \multicolumn{4}{|c|}{$90 \%$} & \multicolumn{4}{|c|}{$95 \%$} \\
\hline & & $\hat{p}$ & $\hat{\theta}$ & $\hat{\lambda}$ & $\hat{k}$ & $\hat{p}$ & $\hat{\theta}$ & $\hat{\lambda}$ & $\hat{k}$ & $\hat{p}$ & $\hat{\theta}$ & $\hat{\lambda}$ & $\hat{k}$ & $\hat{p}$ & $\hat{\theta}$ & $\hat{\lambda}$ & $\hat{k}$ \\
\hline \multirow{3}{*}{$\begin{array}{l}(0.25,0.5 \\
0.75,2.25)\end{array}$} & 30 & 0.0199 & 0.0246 & 0.1238 & 0.0968 & 0.0238 & 0.0374 & 0.1475 & 0.1153 & 0.0144 & 0.0119 & 0.0168 & 0.0260 & 0.0172 & 0.0142 & 0.0182 & 0.0291 \\
\hline & 50 & 0.0065 & 0.0234 & 0.0222 & 0.0076 & 0.0078 & 0.0279 & 0.0264 & 0.0091 & 0.0144 & 0.0119 & 0.0168 & 0.0222 & 0.0172 & 0.0142 & 0.0182 & 0.0264 \\
\hline & 100 & 0.0048 & 0.0192 & 0.0135 & 0.0063 & 0.0057 & 0.0229 & 0.0160 & 0.0075 & 0.0041 & 0.0097 & 0.0103 & 0.0160 & 0.0049 & 0.0115 & 0.0123 & 0.0191 \\
\hline
\end{tabular}

